\title{
Damage identification using wireless structural health monitoring system through smart sensor application
}

\author{
A.A.H.S. Shirazi, F. Mustapha *, K.A. Ahmad
}

Department of Aerospace Engineering, Universiti Putra Malaysia 43400 Serdang, Selangor, Malaysia

\section{A RT ICLE INFO}

\section{Article history:}

Received 9 August 2016

Received in revised form

15 September 2016

Accepted 10 October 2016

\section{Keywords:}

Arduino

Microcontroller

Piezoelectric

PZT Sensor

Structural health monitoring

\begin{abstract}
A B S T R A C T
Delamination, disbanding, void, low impact resistance and visible internal damage are some of damages uniquely found in the structure. Any damage found on the structure require repair. The inspection need to be done to detect the damage on structure before the structure can be repaired. NonDestructive Inspection (NDI) and Structural health monitoring (SHM) concept were used to detect flaws from structures. But the SHM differs from NDT which the system used to monitor the integrity of mechanical structures in a continuous and independent way. SHM helps to reduce financial cost for maintenance. SHM can monitor this situation in active and passive states, either by online or offline monitoring. The use of SHM is to augment the NDI application and not to replace it. This paper presents the damage identification technique by using the improved design of wireless structural health monitoring system. Smart PZT sensors were used as an actuator and receiver, coupled with two XBee's and two Ardiuno as signal generator and signal receiver. Program execution on transmitting and receiving the ultrasonic guided wave via the PZT sensor had been written in Makerplot. The acquired results showed that the wave is more even in non-defected area and disrupted in affected area.
\end{abstract}

(C) 2017 The Authors. Published by IASE. This is an open access article under the CC BY-NC-ND license (http://creativecommons.org/licenses/by-nc-nd/4.0/).

\section{Introduction}

Failure and damage in the structural design and system will caused a million dollar damage and probably losing of life. Although the careful design, periodic inspection and maintenance of structural system has been done, the unexpected damage still can be happened because uncertainties in the structure design and environmental factor (Weng et al., 2008). By definition, the damage is the changes on the structural and mechanical system by comparing the undamaged state and initial states which affect the current and future performance of the system (Farrar et al., 2001). This can be limited to the changes in material behavior or geometric properties or both depending on the component specification (Boller and Meyendorf, 2008).

Since last decades, the maintenance strategies has been evolved and growth. Prognostics and health management which includes SHM is the latest strategies and has been recognized in high

\footnotetext{
* Corresponding Author.

Email Address: faizalms@upm.edu.my (F. Mustapha) https://doi.org/10.21833/ijaas.2017.02.007

2313-626X/C 2017 The Authors. Published by IASE.

This is an open access article under the CC BY-NC-ND license

(http://creativecommons.org/licenses/by-nc-nd/4.0/)
}

technology community to maintain the assets (Bond et al., 2011). SHM can be defined as the monitoring system for detecting the changes in structural properties which can effects the performance and safety of the main system, building or plant. SHM provides monitoring result on time scale of the damage and the severity of the damage (Kim et al., 2007). The changes of properties can be suddenly happened such in earthquake, typhoon or rough wind or can be slowly effect the material properties due to corrosion, fatigue and others.

The system has capability to diagnose and prognosis the failure using online system. Life remaining for the critical structural can be predicted and necessary action can be taken by maintenance engineer (Keller and Ray, 2003).

The diagnostics result from the SHM provides the information on the current state of structure for prognostics. The damage evolution and Remaining Useful Life (RUL) can be identified and maintenance can be performed depending on the current status. The cycle of the diagnostic and prognostics can be summarized at Fig. 1 (Achenbach, 2009).

In this method, the measurement instrument will provide the data on the condition of material on the particular time. The data will be analyzed and the evaluation will provide the report on the current 
condition of degradation material. The material condition will provide the basis information for the engineers to decide either the component need to proceed for repair or the plant can be resume operation as usual.

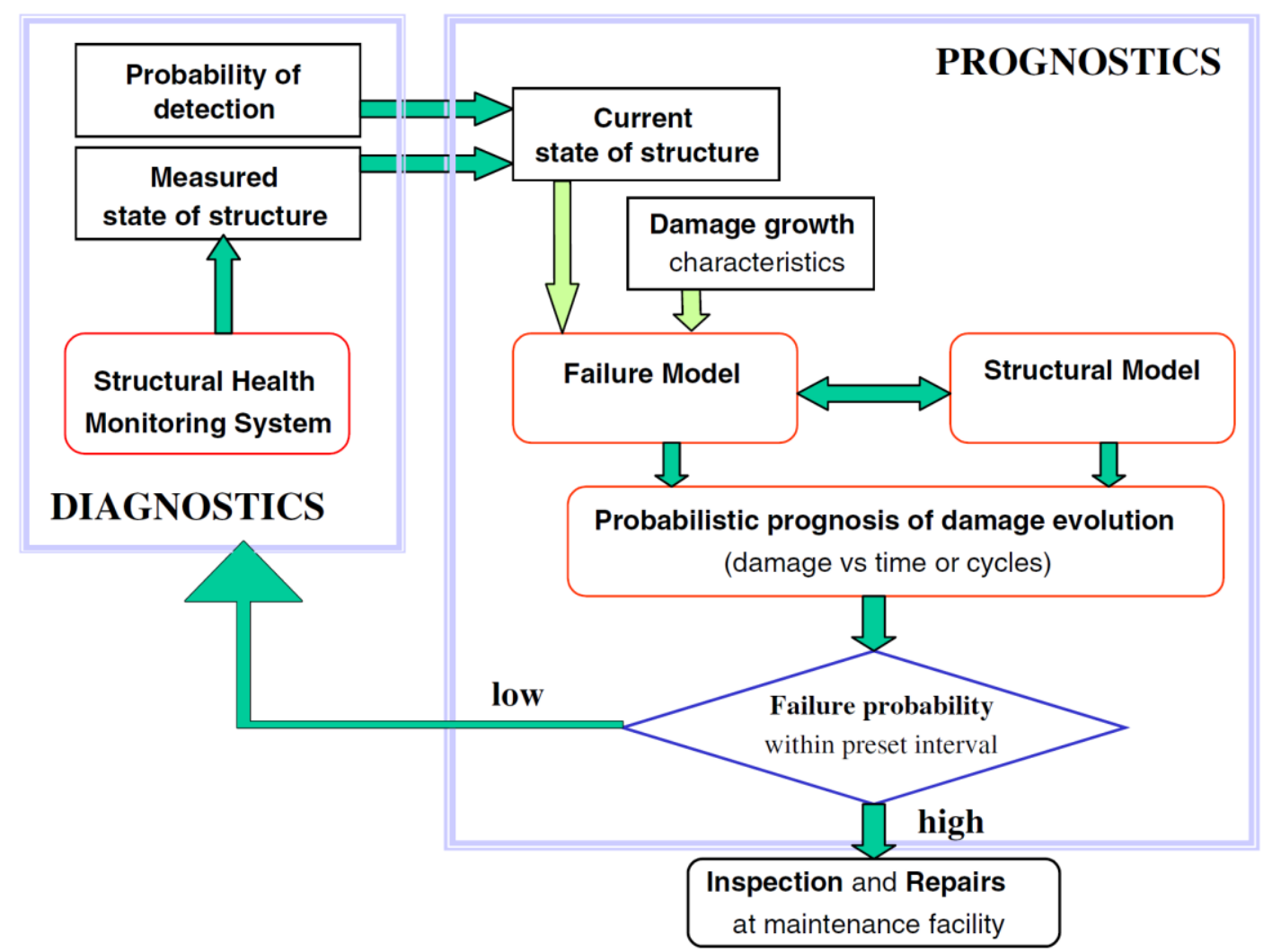

Fig. 1: The cycle of diagnostic and prognostic for SHM (Achenbach, 2009)

\section{Damage detection technique}

The main objective of damage detection techniques is to detect any anomalies from the baseline pattern, which are the indications of damage presence (Jardine et al., 2006). The degree of damage has an immediate or prolonged effect on a structure.

Damage identification involves five closely related disciplines that include Structural Health Monitoring (SHM), Non-Destructive Evaluation (NDE), Condition Monitoring (CM), Damage Prognosis (DP) and Statistical Process Control (SPC). Normally, SHM is related with on-line, autonomous global damage identification in structural systems. $\mathrm{CM}$ is quite similar to SHM, but addresses damage identification in rotating and reciprocating machinery. Meanwhile, NDE is frequently carried out off-line in a local manner with some knowledge of the damage location. SPC is a process-based rather than structure-based. It uses a wide range of sensors to monitor changes in a process, one cause of which can result from structural damage. However, numerous statistical monitoring tools developed for SPC have been adapted to CM and SHM applications.

When damage can be detected at its earliest possible stage and an immediate response takes place, it can significantly reduce the maintenance cost. However, it is not the ideal case to detect damage at its early stage. Previous maintenance philosophy is based on hard time (HT) in order to prevent any catastrophic events. Parts are removed and replaced within the stipulated time or after certain usage cycles. The schedule is based on the theoretical life retrieved from a fatigue test with an added safety factor (Ghobbar and Friend, 2002). This leads to good parts being removed and contributes to the high cost of operation. In order to save cost, selected parts, especially the rotating machinery, were put under condition monitoring (Elforjani et al., 2012). The parts were allowed to operate until anomalies were detected. In terms of structure, the components were put on non-destructive inspection (NDI) after a certain interval or any damage emerged (Wu and Syau, 1993).

However, these two systems require the part or component to be shut down for inspection. This is known as down time and delays operation, which leads to loss of profit. The longer the downtime, the more revenue is lost. The new philosophy is to actively monitor the component, which is known as Structural Health Monitoring or SHM (Worden and Manson, 2007). This system allows monitoring in offline, online, active and passive modes. Fig. 2 shows the evolution of damage detection systems.

\section{Structural health monitoring (SHM)}

The process of implementing a damage detection strategy for aerospace, civil, and mechanical engineering infrastructure is referred to as Structural Health Monitoring (SHM). Usage 
monitoring (UM) attempts to measure the inputs to and responses of a structure before damage so that regression analysis can be used to predict the onset of damage and deterioration in structural condition. Prognosis is the coupling of information from SHM, UM, current environmental and operational conditions, previous component and system level testing, and numerical modelling to estimate the remaining useful life of the system.

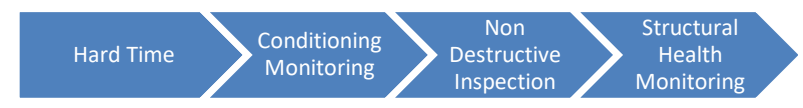

Fig. 2: The evolution of damage detection techniques (Worden and Manson, 2007)

The SHM process involves the observation of a system over time using periodically sampled dynamic response measurements from an array of sensors, the extraction of damage-sensitive features from these measurements, and the statistical analysis of these features to determine the current state of the system's health.

For long-term SHM, the output of this process is periodically updated information regarding the ability of the structure to perform its intended function in light of the inevitable aging and degradation resulting from operational environments. After extreme events, such as earthquakes or blast loading, SHM is used for rapid condition screening and aims to provide, in near real time, reliable information regarding the integrity of the structure.

Structural health monitoring (SHM) is one of key multidisciplinary areas in monitoring and checking damage on the material structure. The remaining multidisciplinary areas are known as condition monitoring (CM), non-destructive evaluation (NDE) and statistical process control (SPC).

SHM is a progression involving technical data acquisition, validation and analysis in order to assist the life-cycle management decisions (Hall and Conquest, 1999). SHM also define as the process with the capability of monitoring the condition of structure and able to detect any damage occurs in the structure itself for a period of time (Catbas et al., 2008). The damage characterization is being described as any changes or alteration in material or structure which caused failure to perform at designed condition and eventually shortened designed operation life (Hall and Conquest, 1999).

Other definition of SHM is the measurement of structure at its operating and loading environment which any sign of operational incidents, anomalies or damages can be followed accessed appropriately using critical responses so that ensure the serviceability, safety reliability and smooth operation. Hence, SHM is checking process that constantly detects the changes in the structure during its lifetime in order to have improvement on the reliability of the structure and to lessen the life cycle cost (Mustapha et al., 2005).
Both of the damage detection techniques which are conditioning monitoring (CM) and Non Destructive Inspection (NDI) have been used for quite some time. However they are suitable for rotating machinery and structural integrity assessment respectively. SHM combines both damage detection philosophies and translates them into an active/passive of online/offline systems in order to monitor the health status of the structure (Farrar et al., 2007) as shown in Fig. 3.

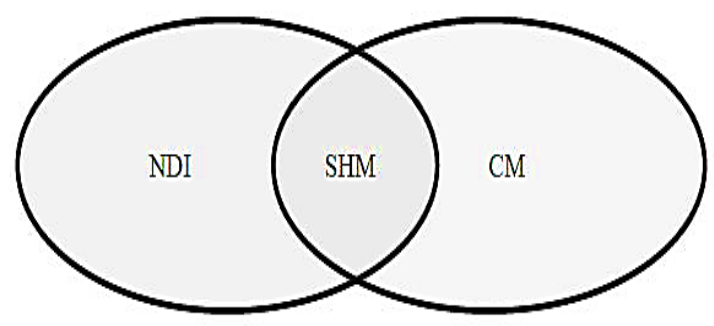

Fig. 3: SHM philosophy (Farrar et al., 2007)

The advancement in sensor technology and data acquisition apparatus has made this approach feasible as the related sensors are becoming smaller and computing power is increasing respectively. Since the sensors are small, they can be embedded or on-surface installed without giving a tremendous weight penalty. The main aircraft manufacturers, such as Boeing (Benedettini et al., 2009) and Airbus, are gearing toward implementing this technology as the material structures become more advanced.

However, most of the SHM damage detection systems, using various methods and sensor technologies, mainly assess the state of the parent structure. The health or condition is evaluated because the response behaves differently when any damage exists. During the operation of the aircraft, the external structures are exposed to the environmental conditions, whereas the internal structures are exposed to the system within the aircraft. Both types of damage, if undetected during the walk-around check or during the maintenance visit, may lead to catastrophic events

Any structure that has SHM system set up is regards as full scale experimental model and system. Through this set up, the condition of the structure can be predicted by observing the recorded loads and responses during the experiment. Basically, structural health monitoring system includes four major components which are sensors, data acquisition and transmission systems, database for effective data management and health diagnosis (Liu et al., 2011). Data processing, damage detection and reliability analysis are the elements fall under health diagnosis program.

In general, these benefits of using SHM are to reduce the maintenance costs and to increase the availability of the structures (Achenbach, 2009). The advantages of SHM had been summarized comparing to schedule-based NDE from oil and gas industries point of view. The advantages are listed as below: 
- Quick assessment of possible damage incidents

- Check or assessment of materials used

- Reduced life cycle total ownership costs

- Increase on safety (Achenbach, 2009).

\section{Hardware and software}

The board system will used the open platform Arduino Uno board where the system embedded with ATMega328 chip as processing controller. It has analog and digital I/O and with friendly programming features that have flexibility for the non-electronic engineers to create the prototype system with user friendly hardware and software programs. The operating voltage is between 5 to 12 $\mathrm{V}$ and has $32 \mathrm{~KB}$ memory which is enough for programming purposes. The system is open source system platform where the board and programming software is under licensed with Creative Common License.

The wireless hardware will used XBee to transmit the data from arduino to the computer. XBee made by Digi International is the popular radio communication module to transfer the data from the board to the data acquisition module. There are 3 frequencies range $(2.4 \mathrm{GHz}, 902-928 \mathrm{MHz}$ and 865 - $868 \mathrm{MHz}$ ) for data transmission. XBee has capability to program wireless point to point and point to multipoint communication protocol. To couple up with Arduino, XBee shield is required as attachment to the board and XBee will be mounted at the top of the shield. The shield and XBee have to be programmed using XCTU software before the transmission of data to the computer through the USB XBee Explorer can be performed. XBee explorer has to be mounted on the USB port with XBee wireless module attached for wireless transmission and reception.

Computer will be used as data storage and mining with the XBee wireless and explorer mounted on the USB port. The data can be view using serial monitor features on the arduino program software. The arduino software will collect data from the serial COM port that specify by the computer and display the data in real time. There is also other software that has capability to visualize the data using graph in real time mode and on this project Maker Plot has been chosen for data visualization.

Maker Plot developed by Selma Solutions is the software that has capability to acquire the data from the serial connection. The connection either by wired or wireless can be graphically display using the software. The software has capability to plot the digital and analog data with monitoring data features. The Graphical User Interface of the software is user friendly and has multiple capabilities to perform the required job. The software is compatible with arduino, Basic Stamp and any other microcontroller board with serial data capability.

\section{Experimental procedures}

Circuit of the transmitter and receiver are based on Arduino Cookbook and ARDX Guide Book. The wiring circuit of the transmitter and receiver were shown in Fig. 4 and Fig. 5.

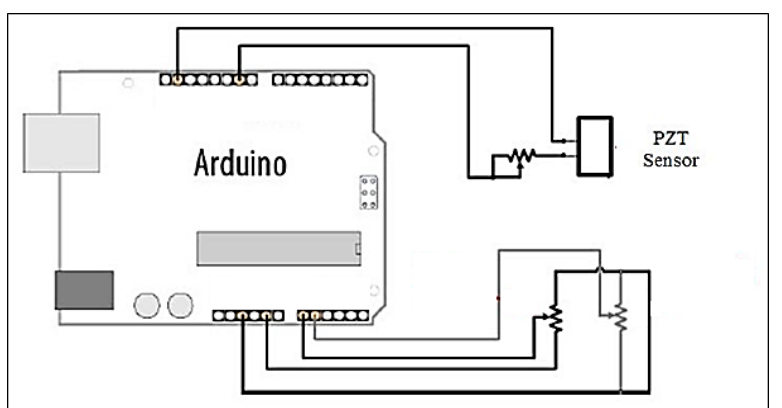

Fig. 4: Circuit for transmitting the frequency

The analogue input from variable resistor is to adjust the frequency. Based on the program, the frequency maximum is $196.2 \mathrm{kHz}$ and the resistor will adjust to maximum for best setting. The PIC 155 wiring connected to the pin 9 as digital output. The preset program will provide the voltage frequency in bit to the PIC 155. The characteristics of PIC 155 will generate the required frequency and produce the wave propagation in the material.

The transmitter circuit produces the electrical signal based on program and the frequency was translated by the PIC 155 to the wave. The wave will conducted through the material and receiver will detect the frequency.

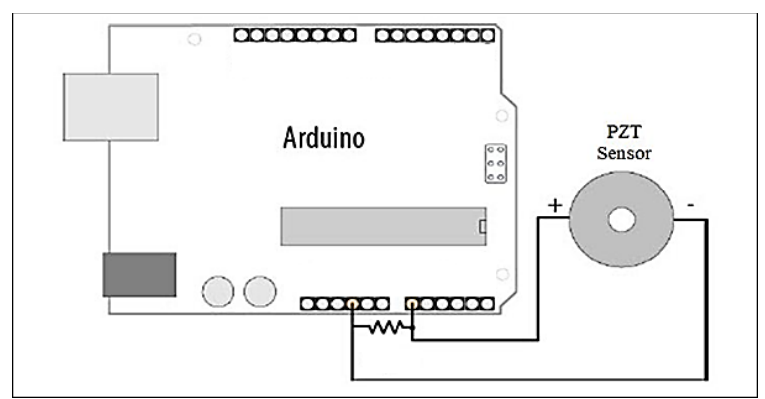

Fig. 5: Circuit for receiving the frequency

The Piezo Sensor mechanical actuation will be translated to the voltage frequency. The processing Arduino Board process the voltage to the bit data $(0$ to 1023 bit $=0$ to $5 \mathrm{~V}$ ) (38). The bit data will be transfer to the XBee transmitter and the data will be sent to the XBee receiver. In this experiment, the XBee module has been setup in Point to Point configuration. The receiver in the serial communication will received the data and the computer will plot the data using the MakerPlot software installed on the receiver computer. The plotted data represent the frequency in bit versus time. The block diagram and system setup has been shown in Fig. 6 and Fig. 7.

By referring to the experimental setup in Fig. 7 above, the PIC 155 is placed on the specimen with $100 \mathrm{~mm}$ distance between transmitter and receiver. 
The experiment test conducted with two conditions (Figs. 8 and 9).

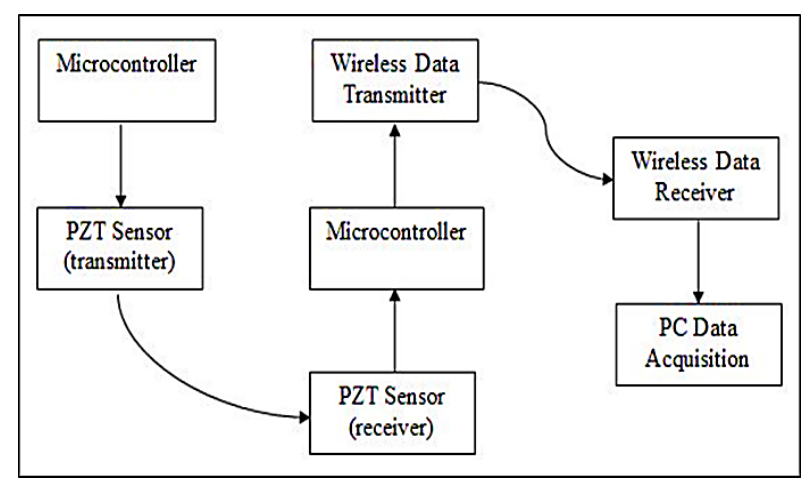

Fig. 6: Block diagram for wireless SHM system

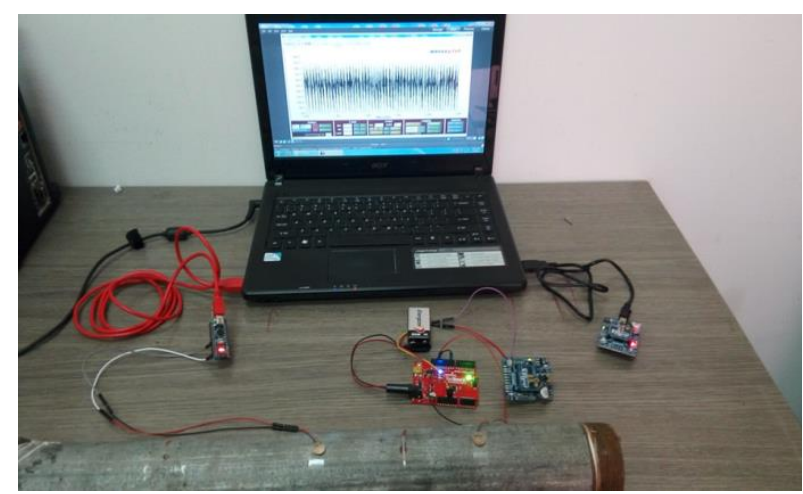

Fig. 7: Experimental setup for the wireless SHM system

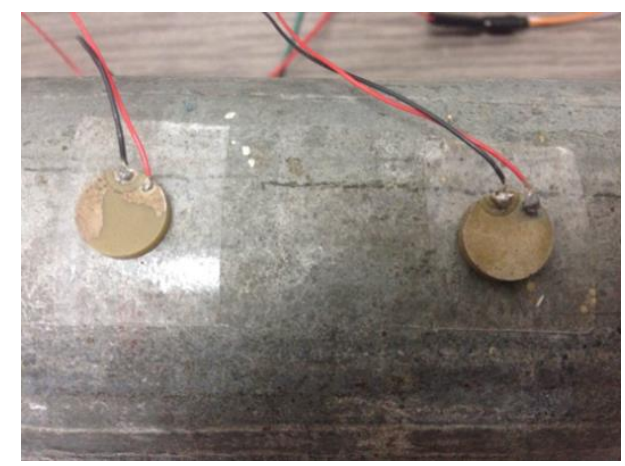

Fig. 8: Testing on undamaged specimen

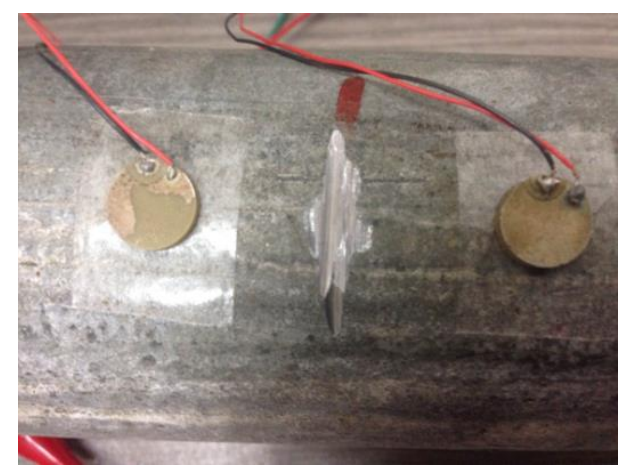

Fig. 9: Testing on damaged specimen

The experimental test setup is to show that the wireless SHM system that has been developed has possibility to represent comparison between undamaged specimen and damaged specimen. The comparison of the data before and after the damage on the material will be represented on next section.

\section{Result analysis}

The setup using the Arduino system has been successfully provides the wireless data to the computer. The MakerPlot software shows the plotted data for damaged and undamaged specimens in real time. The plotted data for undamaged and damaged specimens are shown at Fig. 10 and Fig. 11.

Successful plotted data showed that there are significant results on the undamaged specimen and damaged specimen. By comparison on both plotted data, the wireless SHM system shows the undamaged material data has a peak up to 15 bit and the data is fluctuated in every second. But for the damage data, the highest fluctuation is 2 bit with seldom fluctuating. The comparison of plotted graph has been represented in Fig. 12.

In undamaged specimen, the frequency is absorbed directly to the receiver sensor without any disturbance but for damage specimen, there is disturbance on wave flow inside the material. The wave is lost in half way and there is less fluctuation and data received by the transmitter.

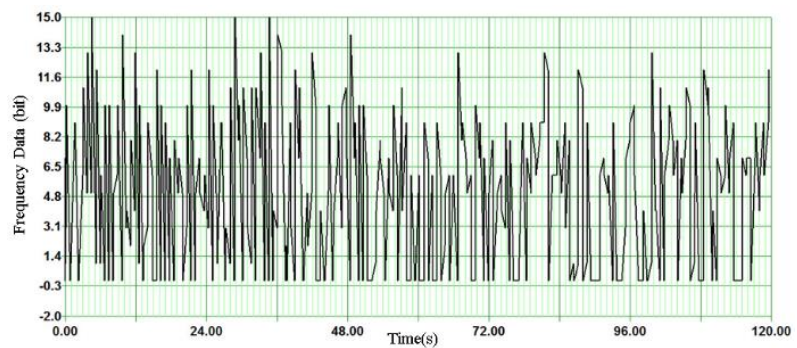

Fig. 10: Undamaged specimen sample data

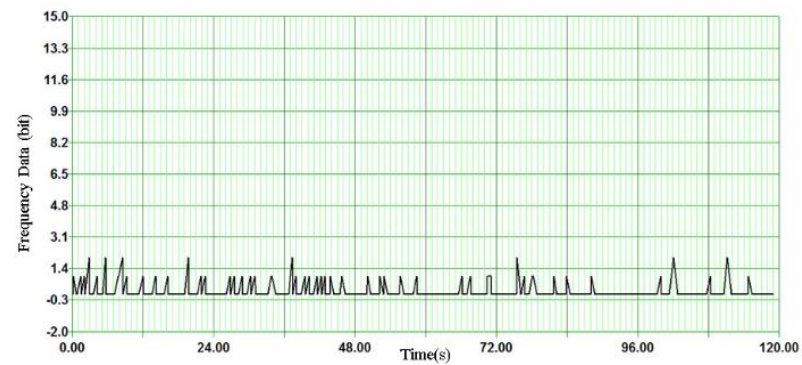

Fig. 11: Damaged specimen sample data

\section{Conclusion}

SHM is the advance technologies that will be used on the current and the next generation of nuclear power plant. The SHM board has been developed and successfully demonstrated and function. The damage has been successfully identified by comparison between damage and undamaged data using Wireless SHM System from this project.

The extended capability with demonstrating other material damage has to be research further before the system can be commercialized. The system potential shall be further investigated and utilized where the Open Platform from Arduino has great capability for the researcher, scientist and innovator to generate creative and useful application for the human use. 


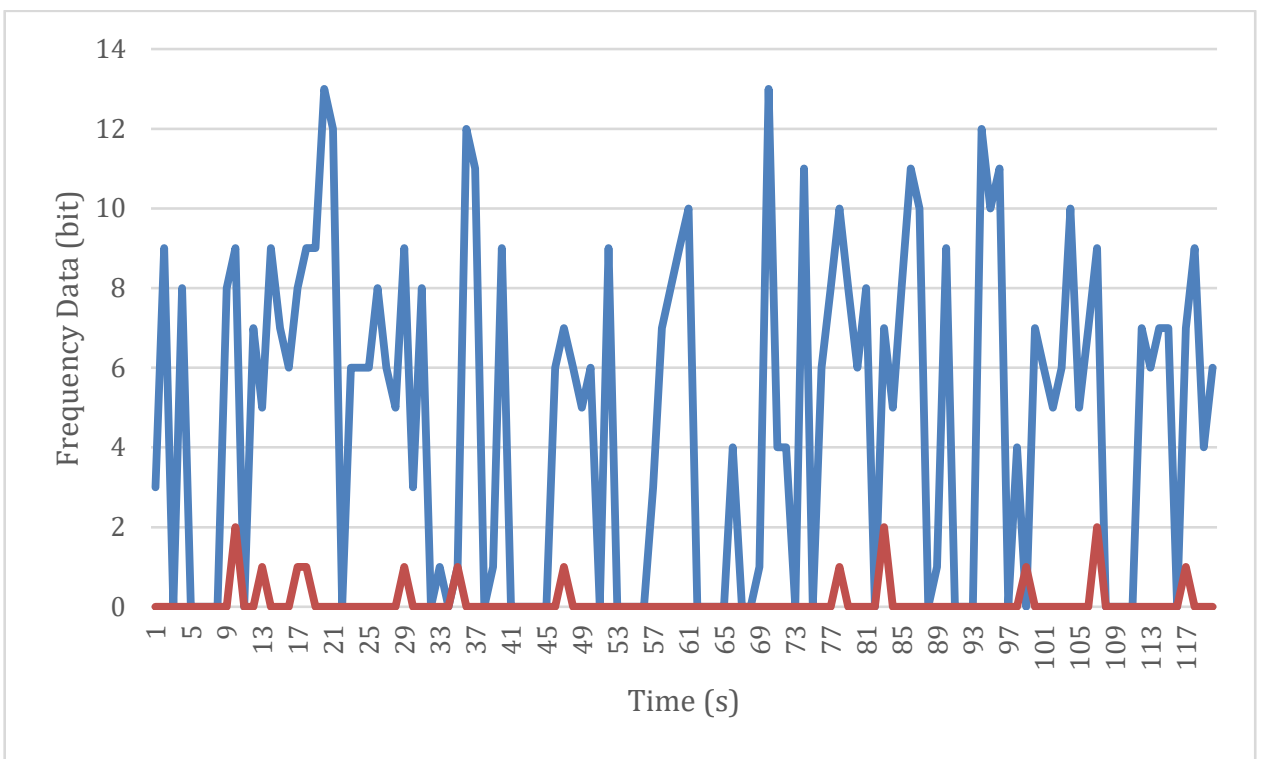

Fig. 12: The plotted graph for damaged and undamaged specimen comparison

\section{References}

Achenbach JD (2009). Structural health monitoring-What is the prescription?. Mechanics Research Communications, 36(2): $137-142$.

Benedettini O, Baines TS, Lightfoot HW, and Greenough RM (2009). State-of-the-art in integrated vehicle health management. Proceedings of the Institution of Mechanical Engineers, Part G: Journal of Aerospace Engineering, 223(2): 157-170.

Boller C and Meyendorf N (2008). State-of-the-art in Structural Health monitoring for aeronautics. In the Proceedings of the International Symposium on NDT in Aerospace, Fürth Germany.

Bond LJ, Ramuhalli P, Tawfik MS, and Lybeck NJ (2011). Prognostics and life beyond 60 years for nuclear power plants. In the IEEE conference on Prognostics and Health Management (PHM), IEEE, Montreal, Canada: 1-7. https://doi.org/10.1109/ICPHM.2011.6024316

Catbas FN, Gul M, and Burkett JL (2008). Conceptual damagesensitive features for structural health monitoring: laboratory and field demonstrations. Mechanical Systems and Signal Processing, 22(7): 1650-1669.

Elforjani M, Mba D, Muhammad A, and Sire A (2012). Condition monitoring of worm gears. Applied Acoustics, 73(8): 859-863.

Farrar CR, Doebling SW, and Nix DA (2001). Vibration-based structural damage identification. Philosophical Transactions of the Royal Society of London A: Mathematical, Physical and Engineering Sciences, 359(1778): 131-149.

Farrar CR, Worden K, Todd MD, Park G, Nichols J, Adams DE, and Farinholt K (2007). Nonlinear system identification for damage detection (No. LA-14353-MS). Los Alamos National Laboratory (LANL), Los Alamos, USA.

Ghobbar AA and Friend CH (2002). Sources of intermittent demand for aircraft spare parts within airline operations. Journal of Air Transport Management, 8(4): 221-231.
Hall SR and Conquest TJ (1999). The total data integrity initiative-structural health monitoring, the next generation. In the Proceedings of the USAF ASIP Conference, $2^{\text {nd }}$ Edition.

Jardine AK, Lin D, and Banjevic D (2006). A review on machinery diagnostics and prognostics implementing condition-based maintenance. Mechanical Systems and Signal Processing, 20(7): 1483-1510.

Keller E and Ray A (2003). Real-time health monitoring of mechanical structures. Structural Health Monitoring, 2(3): 191-203.

Kim S, Pakzad S, Culler D, Demmel J, Fenves G, Glaser S, and Turon $M$ (2007). Health monitoring of civil infrastructures using wireless sensor networks. In the $6^{\text {th }}$ International Symposium on Information Processing in Sensor Networks (IPSN '07), IEEE, Massachusetts, USA: 254-263. https://doi.org/10.1109/ IPSN.2007.4379685

Liu F, Mao Z, and Su W (2011). Outlier detection for process control data based on a non-linear Auto-Regression Hidden Markov Model method. Transactions of the institute of measurement and Control, 34(5): 527-538.

Mustapha F, Manson G, Pierce SG, and Worden K (2005). Structural health monitoring of an annular component using a statistical approach. Strain, 41(3): 117-127.

Weng JH, Loh CH, Lynch JP, Lu KC, Lin PY, and Wang, Y (2008). Output-only modal identification of a cable-stayed bridge using wireless monitoring systems. Engineering Structures, 30(7): 1820-1830.

Worden K and Manson G (2007). The application of machine learning to structural health monitoring. Philosophical Transactions of the Royal Society of London A: Mathematical, Physical and Engineering Sciences, 365(1851): 515-537.

Wu WF and Syau JJ (1993). Study of the nondestructive inspection frequency based on structural reliability considerations. NDT and E International, 26(5): 249-254. 